\title{
Demonstration of Pick and Place Assembly for Scaled MEMS Devices
}

\author{
Jangbae Jeon, ${ }^{*}$ Igor Gory, ${ }^{* *}$ George Skidmore, ${ }^{* *}$ M.J. Kim, ${ }^{*}$ and B.E. Gnade* \\ * Department of Electrical Engineering, University of Texas at Dallas, 2601 N. Floyd Road, \\ Richardson, Texas 75083 \\ ** Zyvex Corporation, 1321 N. Plano Road, Richardson, Texas 75081
}

The applications of microelectromechanical systems (MEMS) are often limited because of a lack of 3-dimensional assembly techniques. Nano-assembly techniques such as "pick and place" will allow more complex structures to be fabricated, and therefore more applications will become available. In order to overcome these limitations, there is considerable on-going research on different assembly techniques such as self assembly, gripping, smart assembly, and micro-manipulation [1][2].

In this paper we demonstrate a "pick and place" assembly process for scaled MEMS devices. The micromachined end-effectors, parts, and socket which are tethered to the device layer were fabricated on 6 inch silicon-on-insulator (SOI) wafers which have $6 \pm 0.8 \mu \mathrm{m}$ of single crystal silicon (the device layer) on a $2 \mu \mathrm{m}$ thick buried oxide layer, as shown in Fig. 1.

Because the MEMS devices in this study are $10 \times$ smaller than those used in previously reported micro-assembly, scanning electron microscopy using a FEI-Nova NanoLab 200 dual column focused ion beam (FIB) system, which has a secondary electron image resolution of $1.5 \mathrm{~nm}$ at $15 \mathrm{kV}$, is used for alignment instead of an optical microscope. The Zyvex F-100 nano-manipulator, which has 4 degrees of freedom (DOF), physically coupled to the FEI Nova 200 sample stage which has 5 DOF, as shown in Fig. 2 [3], is used for manipulation and assembly.

The "pick and place" process is achieved with the sequence of operations shown in Fig. 3;

1. Insert the tip of the end-effector into the part until the end-effector is secured between the two beams of the part.

2. Move and push the end-effector to the top of the part for de-tethering the part from the die.

3. Lift the gripper from the die by moving the end-effector.

4. Rotate the actuator of the manipulator to make the part perpendicular to the die.

5. Bring the part to the opening of the socket.

6. Insert the tip of the part into the center of the socket.

7. Move the tip of the end-effector down, between the two beams of the part.

8. Slide the tip of the end-effector from the part after the two beams are secured in the socket.

\section{References}

[1] Daniel N. Pascual, Solid State Technology 48 (2005) 38.

[2] H. Van Brussel et al., Annals of the CIRP 49 no.2 (2000) 451.

[3] Kenneth Tsui et al., J. Micromech. Microeng. 14 (2004) 542.

[4] This research is supported in part by the NIST ATP Award 70NANB1H3021, and the UTD Erick Jonsson School of Engineering and Computer Science. 


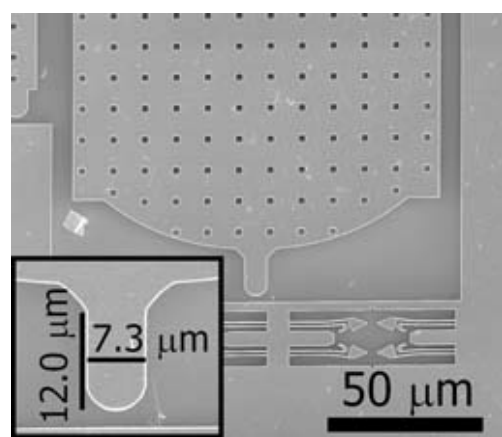

(a)

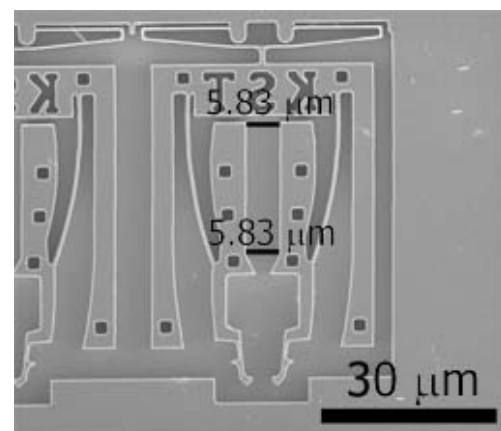

(b)

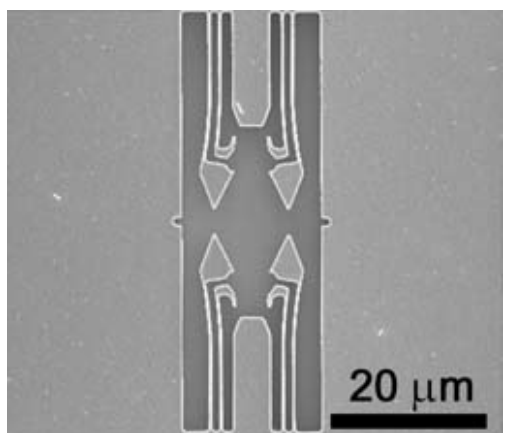

(c)

FIG. 1. SEM images of the micromachined (a) end-effector, (b) part and (c) socket.

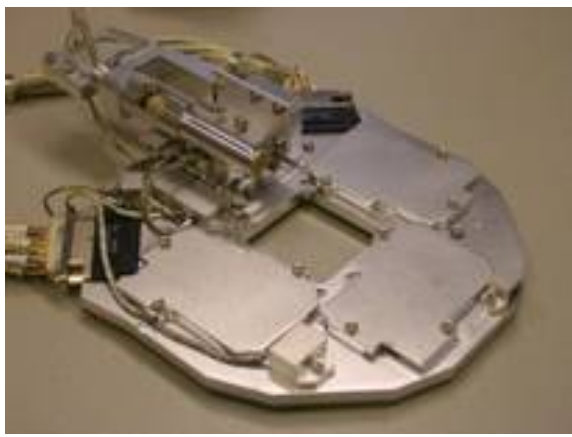

(a)

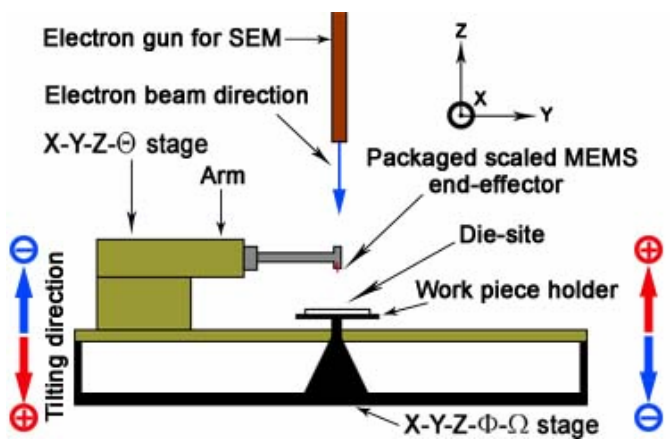

(b)

FIG. 2. (a) The Zyvex F-100 manipulator and (b) diagram of the high precision robotics system used for nano-assembly.

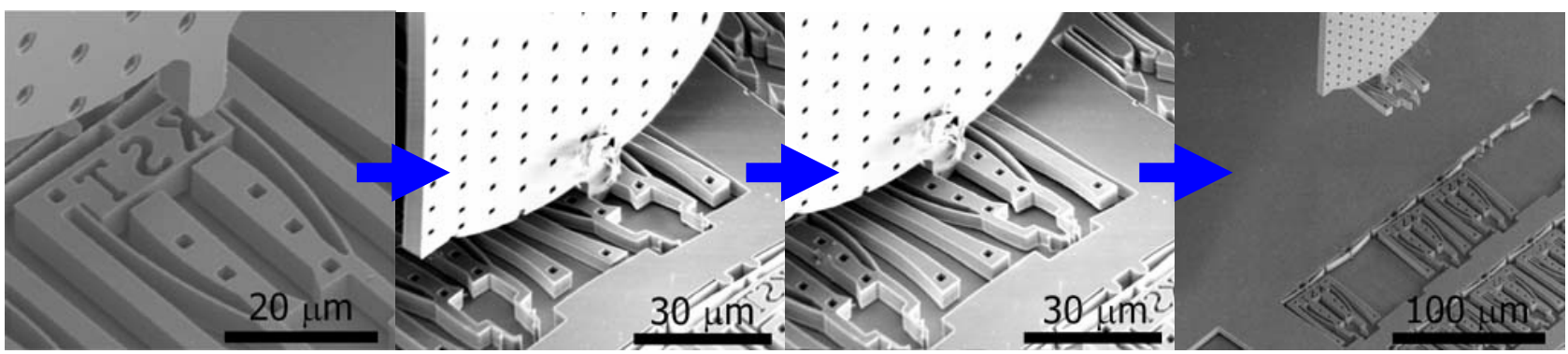

(a)

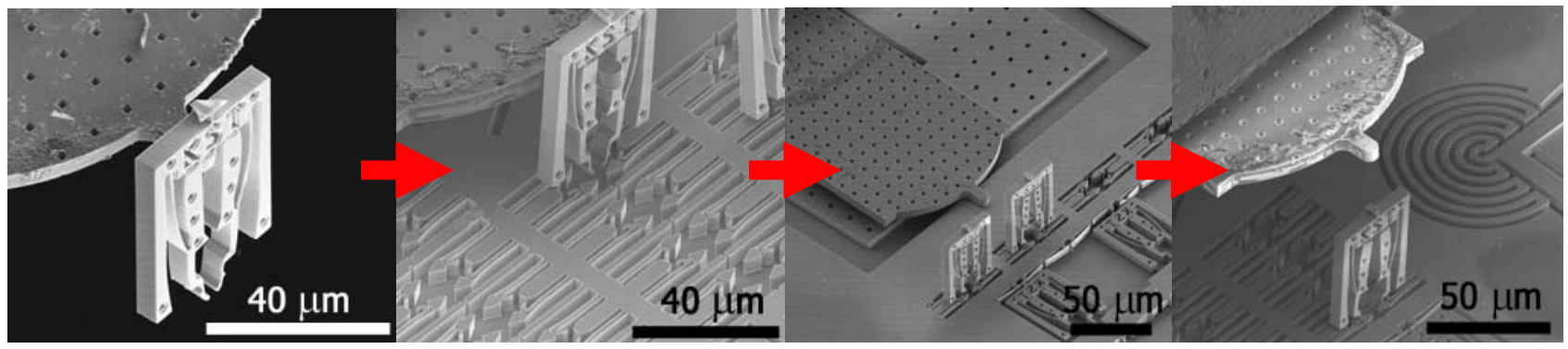

(b)

FIG. 3. Nano-assembly procedure of (a) pick and (b) place process. 